\title{
FROM SIMPLE-MINDED COLLECTIONS TO SILTING OBJECTS VIA KOSZUL DUALITY
}

\author{
HAO SU AND DONG YANG
}

\begin{abstract}
Given an elementary simple-minded collection in the derived category of a nonpositive dg algebra with finite-dimensional total cohomology, we construct a silting object via Koszul duality.
\end{abstract}

MSC 2010 classification: 18E30, 16E45.

Keywords: Silting object, simple-minded collection, non-positive dg algebra, positive $A_{\infty}{ }^{-}$ algebra.

\section{INTRODUCTION}

Projective modules, simple modules and the interaction between them are crucial in the representation theory of finite-dimensional algebras. In the context of triangulated categories, silting objects and simple-minded collections are generalisations of projective modules and simple modules, respectively. Silting objects are 'generators' of bounded co-t-structures ([2, 5, 17]), and simple-minded collections are 'generators' of bounded t-structures whose hearts satisfy the Jordan-Hölder property ([3, 13]).

In [18] (and [19, 13]), Rickard provided a method to construct a silting object of the bounded homotopy category $K^{b}(\operatorname{proj} \Lambda)$ from a given simple-minded collection in the bounded derived category $\mathcal{D}^{b}(\bmod \Lambda)$, where $\Lambda$ is a finite-dimensional algebra. In this paper, we provide an alternative approach to Rickard's construction. Our approach works more generally for nonpositive dg algebras with finite-dimensional total cohomology.

Theorem 1.1. Let $A$ be a non-positive dg algebra over a field $k$ with finite-dimensional total cohomology. For an elementary simple-minded collection $\left\{X_{1}, \ldots, X_{r}\right\}$ of the finite-dimensional derived category $\mathcal{D}_{f d}(A)$, there exists a unique (up to isomorphism) silting object $M=M_{1} \oplus$ $\ldots \oplus M_{r}$ of the perfect derived category $\operatorname{per}(A)$ such that for $1 \leq i, j \leq r$ and $p \in \mathbb{Z}$

$$
\operatorname{Hom}_{\mathcal{D}_{f d}(A)}\left(M_{i}, \Sigma^{p} X_{j}\right)= \begin{cases}k & \text { if } i=j \text { and } p=0, \\ 0 & \text { otherwise. }\end{cases}
$$

The key idea of the construction of $M$ is to use the triangulated version of Koszul duality ([9, 14, 15]). On the Koszul dual side we use $A_{\infty}$-algebras instead of dg algebras. One reason is that in this way the simple modules are easily obtained because the involved $A_{\infty}$-algebras are naturally augmented.

As a consequence of Theorem 1.1, we obtain the following theorem, generalising [13, Theorems 6.1 and 7.12$]$. 
Theorem 1.2. Let $A$ be a non-positive dg algebra over an algebraically closed field with finitedimensional total cohomology. There are one-to-one correspondences which commute with mutations and which preserve partial orders between

(1) equivalence classes of silting objects in $\operatorname{per}(A)$,

(2) isomorphism classes of simple-minded collections in $\mathcal{D}_{f d}(A)$,

(3) bounded t-structures of $\mathcal{D}_{f d}(A)$ with length heart,

(4) bounded co-t-structures of $\operatorname{per}(A)$.

A similar result is claimed by Keller and Nicolás in an unpublished manuscript, with $A$ replaced by a homologically smooth non-positive dg algebra with finite-dimensional cohomology in each degree.

The paper is structured as follows. In Section 2, we recall the basics on silting objects and non-positive dg algebras. In Section 3, we recall the basics on $A_{\infty}$-algebras and $A_{\infty}$-modules. In Section 4, we recall the definition of simple-minded collections and study strictly unital minimal positive $A_{\infty}$-algebras, which are closely related to simple-minded collections. In Section 5 we provide the construction of a silting object from a given simple-minded collection.

Throughout the paper, let $k$ be a field and let $D=\operatorname{Hom}_{k}(?, k)$ be the $k$-dual. Without further remark, modules will be right modules and all categories are $k$-categories.

Acknowledgement. The first-named author is deeply indebted to the second-named author for his guidance and help. The second-named author would like to thank Mark Blume for valuable remarks on an earlier version. He acknowledges support from the National Science Foundation in China No. 11401297 and a project funded by the Priority Academic Program Development of Jiangsu Higher Education Institutions.

\section{Silting OBJECTS AND NON-POSITIVE DG ALGEBRAS}

In this section we recall the definitions and standard facts on silting objects and non-positive dg algebras.

2.1. Silting objects. Let $\mathcal{C}$ be a triangulated category with suspension functor $\Sigma$. For a set $\mathcal{S}$ of objects of $\mathcal{C}$, let $\operatorname{add}(\mathcal{S})=\operatorname{add}_{\mathcal{C}}(\mathcal{S})$ be the smallest full subcategory of $\mathcal{C}$ containing $\mathcal{S}$ and closed under taking direct summands and finite direct sums, and let thick $(\mathcal{S})=\operatorname{thick}_{\mathcal{C}}(\mathcal{S})$ denote the thick subcategory of $\mathcal{C}$ generated by $\mathcal{S}$, i.e. the smallest full subcategory of $\mathcal{C}$ that is closed under taking suspensions, cosuspensions, extensions and direct summands.

An object $M$ of $\mathcal{C}$ is a silting object of $\mathcal{C}$ if

- $\operatorname{Hom}_{\mathcal{C}}\left(M, \Sigma^{p} M\right)=0$ for any $p>0$,

$-\mathcal{C}=\operatorname{thick}(M)$.

Two silting objects $M$ and $M^{\prime}$ of $\mathcal{C}$ are said to be equivalent if $\operatorname{add}(M)=\operatorname{add}\left(M^{\prime}\right)$.

2.2. Non-positive $\mathbf{d g}$ algebras. Let $A$ be a $\operatorname{dg} k$-algebra. Denote by $\mathcal{D}(A)$ the derived category of (right) dg $A$-modules (see [9, 11]), which is a triangulated category with suspension functor $\Sigma$ the shift functor. Let $\operatorname{per}(A)=\operatorname{thick}\left(A_{A}\right)$, the thick subcategory of $\mathcal{D}(A)$ generated by 
$A_{A}$, the free $\operatorname{dg} A$-module of rank 1 , and let $\mathcal{D}_{f d}(A)$ be the full subcategory of $\mathcal{D}(A)$ consisting of $\operatorname{dg} A$-modules whose total cohomology is finite-dimensional. If $A$ is a finite-dimensional $k$-algebra, then a dg $A$-module is exactly a complex of $A$-modules. So $\mathcal{D}(A)=\mathcal{D}(\operatorname{Mod} A)$ and we have canonical triangle equivalences $K^{b}(\operatorname{proj} A) \rightarrow \operatorname{per}(A)$ and $\mathcal{D}^{b}(\bmod A) \rightarrow \mathcal{D}_{f d}(A)$. For two dg $A$-modules $M$ and $N$, let $\mathcal{H o m} A(M, N)$ denote the complex whose degree $p$ component consists of those $A$-linear maps from $M$ to $N$ which are homogeneous of degree $p$, and whose differential takes $f$ to $d_{N} \circ f-(-1)^{|f|} f \circ d_{M}$, where $f$ is homogeneous of degree $|f|$.

A $\operatorname{dg} A$-module $M$ is said to be $K$-projective if $\mathcal{H o m}_{A}(M, N)$ is acyclic for any $\operatorname{dg} A$-module $N$ which is acyclic. Let $M$ and $N$ be $\operatorname{dg} A$-modules. If $M$ is $K$-projective, then there is a canonical isomorphism

$$
\operatorname{Hom}_{\mathcal{D}(A)}(M, N) \cong H^{0} \mathcal{H} \operatorname{om}_{A}(M, N) .
$$

By [9, Theorem 3.1], for any $\operatorname{dg} A$-module $M$ there is a quasi-isomorphism $\mathbf{p} M \rightarrow M$ of $\mathrm{dg}$ $A$-modules with $\mathbf{p} M$ being $K$-projective.

We say that $A$ is non-positive if $A^{p}=0$ vanishes for all $p>0$. A triangulated category is said to be algebraic if it is triangle equivalent to the stable category of a Frobenius category.

Lemma 2.1 ([13, Lemma 4.1]). (a) Let $A$ be a non-positive $d g$-algebra. The free $d g A$ module of rank 1 is a silting object of $\operatorname{per}(A)$.

(b) Let $\mathcal{C}$ be an idempotent complete algebraic triangulated category and let $M \in \mathcal{C}$ be a silting object. Then there is a non-positive $d g$ k-algebra $A$ together with a triangle equivalence $\operatorname{per}(A) \stackrel{\simeq}{\longrightarrow} \mathcal{C}$ which takes $A$ to $M$.

2.3. Cohomologically finite-dimensional non-positive $\mathbf{d g}$ algebras. Let $A$ be a nonpositive $\mathrm{dg} k$-algebra whose total cohomology is finite-dimensional over $k$. Then both $\mathcal{D}_{f d}(A)$ and $\operatorname{per}(A)$ are Krull-Schmidt, since they are Hom-finite, see for example [1, Proposition 4.2 (a)]. Moreover, $\operatorname{per}(A) \subseteq \mathcal{D}_{f d}(A)$ and $\operatorname{thick}\left(D\left({ }_{A} A\right)\right) \subseteq \mathcal{D}_{f d}(A)$. There is a triangle functor $\nu: \mathcal{D}(A) \rightarrow \mathcal{D}(A)$ (called the Nakayama functor) which restricts to a triangle equivalence $\nu: \operatorname{per}(A) \rightarrow \operatorname{thick}\left(D\left({ }_{A} A\right)\right)$. We have the Auslander-Reiten formula

$$
D \operatorname{Hom}(M, N) \cong \operatorname{Hom}(N, \nu(M))
$$

for $M \in \operatorname{per}(A)$ and $N \in \mathcal{D}(A)$. See [9, Section 10].

Recall from the preceding subsection that $A_{A}$ is a silting object of $\operatorname{per}(A)$. Let $M$ be a silting object of $\operatorname{per}(A)$. We may assume that $M$ is $K$-projective and form the dg endomorphism algebra $\mathcal{E} n d_{A}(M):=\mathcal{H} \operatorname{om}_{A}(M, M)$. Then by [9, Lemma 6.1], we have a derived equivalence $\mathcal{D}\left(\mathcal{E} n d_{A}(M)\right) \rightarrow \mathcal{D}(A)$ taking $\mathcal{E} n d_{A}(M)$ to $M$. Since $H^{p} \mathcal{E} n d_{A}(M)=\operatorname{Hom}_{\mathcal{D}(A)}\left(M, \Sigma^{p} M\right)$, it follows that $\mathcal{E} n d_{A}(M)$ has finite-dimensional total cohomology and $H^{p} \mathcal{E} n d_{A}(M)=0$ for all $p>0$. The subcomplex $\sigma^{\leq 0} \mathcal{E} n d_{A}(M)$, where $\sigma^{\leq 0}$ is the standard truncation in degree 0 , is a dg subalgebra of $\mathcal{E} n d_{A}(M)$. In particular, it is a non-positive dg algebra with finite-dimensional total cohomology. Moreover, the canonical embedding $\sigma^{\leq 0} \mathcal{E} n d_{A}(M) \rightarrow \mathcal{E} n d_{A}(M)$ is a quasiisomorphism of dg algebras, inducing a derived equivalence

$$
\mathcal{D}\left(\sigma^{\leq 0} \mathcal{E} n d_{A}(M)\right) \rightarrow \mathcal{D}(A)
$$


taking $\sigma^{\leq 0} \mathcal{E} n d_{A}(M)$ to $M$. We call $\sigma^{\leq 0} \mathcal{E} n d_{A}(M)$ the truncated $d g$ endomorphism algebra of $M$.

\section{3. $A_{\infty}$-Algebras And $A_{\infty}$-Modules}

In this section we recall the definition and basic properties of $A_{\infty}$-algebras and $A_{\infty}$-modules. We follow [14] and also refer to [10] and [15].

3.1. $A_{\infty}$-algebras. Let $R$ be a separable semi-simple $k$-algebra. An $A_{\infty}$-algebra $A$ over $R$ is a graded $R$-bimodule endowed with a family of homogeneous $R$-bilinear maps $m_{n}: A^{\otimes_{R}} \rightarrow$ $A(n \geq 1)$ of degree $2-n$, called the multiplications of $A$, which satisfy the following identities

$$
\sum_{i+j+l=n}(-1)^{i j+l} m_{i+1+l}\left(\mathrm{id}^{\otimes i} \otimes m_{j} \otimes \mathrm{id}^{\otimes l}\right)=0, n \geq 1 .
$$

Here $i, l \geq 0$ and $j \geq 1$. (In [14, Définition 1.2.1.1], $A_{\infty}$-algebras are defined over a monoidal category $\mathbf{C}$. The above definition is obtained by taking $\mathbf{C}$ as the category of $R$-bimodules.) We are mainly interested in the case when $R$ is a finite direct product of copies of $k$, which we use 'as if it were non-commutative', namely, we do not require that the left and right graded $R$-module structures on $A$ coincide (compare e.g. [16, Section 2.1]). Let $A$ be an $A_{\infty}$-algebra over $R$. $A$ is said to be strictly unital if there is a $R$-bilinear map $\eta: R \rightarrow A$ (called the unit of $A$ ) which is homogeneous of degree 0 such that $m_{n}(\mathrm{id} \otimes \cdots \otimes \mathrm{id} \otimes \eta \otimes \mathrm{id} \otimes \cdots \otimes \mathrm{id})=0$ for $n \neq 2$ (here $\eta$ can occur in any position in the tensor product) and $m_{2}(\mathrm{id} \otimes \eta)=m_{2}(\eta \otimes \mathrm{id})=\mathrm{id}$. Note that the identity (3.1) for $n=1$ is $m_{1}^{2}=0$, thus $A$ is a complex of $R$-bimodules with differential $m_{1}$. $A$ is said to be minimal if $m_{1}=0$. In this case, $A$ is a graded algebra over $R$ with $m_{2}$ as multiplication.

Let $A$ and $B$ be two strictly unital $A_{\infty}$-algebras over $R$. A strictly unital $A_{\infty}$-morphism $f: A \rightarrow B$ of strictly unital $A_{\infty}$-algebras is a family of homogeneous $R$-bilinear maps $f_{n}$ : $A^{\otimes_{R} n} \rightarrow B(n \geq 1)$ of degree $1-n$, such that $f_{1} \eta_{A}=\eta_{B}, f_{n}\left(\mathrm{id} \otimes \cdots \otimes \mathrm{id} \otimes \eta_{A} \otimes \mathrm{id} \otimes \cdots \otimes \mathrm{id}\right)=0$ for all $n \geq 2$, and that

$$
\sum_{i+j+l=n}(-1)^{i j+l} f_{i+1+l}\left(\mathrm{id}^{\otimes i} \otimes m_{j} \otimes \mathrm{id}^{\otimes l}\right)=\sum_{\substack{1 \leq p \leq n \\ i_{1}+\ldots+i_{p}=n}}(-1)^{\omega} m_{p}\left(f_{i_{1}} \otimes \cdots \otimes f_{i_{p}}\right), n \geq 1 .
$$

Here $j \geq 1 ; i, l \geq 0$ and $\omega=\sum_{2 \leq u \leq p}\left(1-i_{u}\right) \sum_{1 \leq v \leq u} i_{v}$. It follows that $f_{1}$ is a chain map with respect to the differentials $m_{1}$. If $f_{1}$ is a quasi-isomorphism of complexes, we say that $f$ is an $A_{\infty}$-quasi-isomorphism. If $f_{n}=0$ for $n \geq 2$, then the above identities amounts to saying that $f_{1}: A \rightarrow B$ commutes with all multiplications $m_{n}$. In this case, we say that $f$ is strict and identify $f$ with $f_{1}$.

Let $A$ be a strictly unital $A_{\infty}$-algebra over $R$. $A$ is said to be augmented if there is a strict $A_{\infty}$-morphism $\varepsilon: A \rightarrow R$ of strictly unital $A_{\infty}$-algebras, which is called the augmentation of $A$. Here we view $R$ as a strictly unital $A_{\infty}$-algebra over $R$ with $m_{2}=\mathrm{id}, m_{n}=0$ for $n \neq 2$ and $\eta=$ id. Let $A$ and $B$ be two augmented $A_{\infty}$-algebras over $R$. An $A_{\infty}$-morphism $f: A \rightarrow B$ of augmented $A_{\infty}$-algebras is a strictly unital $A_{\infty}$-morphism of strictly unital $A_{\infty}$-algebras such that $\varepsilon_{B} f_{1}=\varepsilon_{A}$. 
3.2. Enveloping $\mathrm{dg}$ algebras. A $\mathrm{dg}$ algebra $A$ over $R$ is a $\mathrm{dg} k$-algebra together with a homomorphism $\eta: R \rightarrow A$ of dg $k$-algebras. It can be considered as a strictly unital $A_{\infty^{-}}$ algebra over $R$ with $m_{1}$ being the differential, $m_{2}$ being the multiplication and $m_{n}=0$ for $n \geq 3$.

Theorem 3.1 ([14, Proposition 7.5.0.2] and [14, Lemme 2.3.4.3]). Let $A$ be a strictly unital $A_{\infty}$-algebra over $R$. Then there is a dg algebra $A^{\prime}$ (called a $\mathrm{dg}$ model of $A$ ) over $R$ with a strictly unital $A_{\infty}$-quasi-isomorphism $A \rightarrow A^{\prime}$. If $A$ is augmented over $R$, then $A^{\prime}$ can be taken augmented over $R$ (in this case $A^{\prime}$ is called the enveloping $\mathrm{dg}$ algebra of $A$ ) and the $A_{\infty}$-quasi-isomorphism above is an $A_{\infty}$-quasi-isomorphism of augmented $A_{\infty}$-algebras over $R$.

3.3. $A_{\infty}$-modules. Let $A$ be a strictly unital $A_{\infty}$-algebra over $R$. A (right) $A_{\infty}$-module over $A$ is a graded right $R$-module $M$ endowed with a family of homogeneous $R$-linear maps $m_{n}^{M}$ : $M \otimes_{R} A^{\otimes_{R} n-1} \longrightarrow M(n \geq 1)$ of degree $2-n$ such that (some of the superscripts $M$ on $m$ are omitted)

$$
\sum_{i+j+l=n}(-1)^{i j+l} m_{i+1+l}\left(\mathrm{id}^{\otimes i} \otimes m_{j} \otimes \mathrm{id}^{\otimes l}\right)=0 .
$$

Here $i, l \geq 0$ and $j \geq 1$. The graded $R$-module $M$ equipped with $m_{1}^{M}$ becomes a complex. An $A_{\infty}$-module $M$ is said to be minimal if $m_{1}^{M}=0$. It is said to be strictly unital if $m_{n}^{M}\left(\mathrm{id}_{M} \otimes\right.$ id $\otimes \cdots \otimes$ id $\otimes \eta \otimes$ id $\otimes \cdots \otimes$ id $)=0$ for all $n \geq 3$, and $m_{2}^{M}\left(\operatorname{id}_{M} \otimes \eta\right)=\mathrm{id}_{M}$. If $M^{\prime}$ is a graded $R$-submodule of $M$ such that $m_{n}^{M}$ restricts to $M^{\prime}$ for all $n \geq 1$, then $M^{\prime}$ together with the restriction of $m_{n}^{M}$ is called a submodule of $M$. A together with its multiplications is an $A_{\infty}$-module over $A$. An element $e$ of $A$ is called a strict idempotent if $e \in \operatorname{im}(\eta), m_{2}(e \otimes e)=e$ and for all $n \neq 2$ we have $m_{n}\left(a_{1} \otimes \cdots \otimes a_{n}\right)=0$ if one of $a_{1}, \ldots, a_{n}$ is $e$. If $e$ is a strict idempotent of $A$, then $e A=\left\{e a:=m_{2}(e \otimes a) \mid a \in A\right\}$ is an $A_{\infty}$-submodule of $A$, because

$$
m_{n}\left(e a_{1} \otimes a_{2} \otimes \cdots \otimes a_{n}\right)=e m_{n}\left(a_{1} \otimes a_{2} \otimes \cdots \otimes a_{n}\right) .
$$

Let $M$ and $M^{\prime}$ be two strictly unital $A_{\infty}$-modules over $A$. A strictly unital $A_{\infty}$-morphism $f: M \rightarrow M^{\prime}$ is a family of homogeneous $R$-linear maps $f_{n}: M \otimes_{R} A^{\otimes_{R} n-1} \longrightarrow M^{\prime}(n \geq 1)$ of degree $1-n$ such that $f_{n}\left(\mathrm{id}_{M} \otimes \mathrm{id} \otimes \cdots \otimes \mathrm{id} \otimes \eta \otimes \mathrm{id} \otimes \cdots \otimes \mathrm{id}\right)=0$ for all $n \geq 2$ and that the following identity holds for all $n \geq 1$

$$
\sum_{i+j+l=n}(-1)^{i j+l} f_{i+1+l}\left(\mathrm{id}^{\otimes i} \otimes m_{j} \otimes \mathrm{id}^{\otimes l}\right)=\sum_{s+t=n} m_{1+t}\left(f_{s} \otimes \mathrm{id}^{\otimes t}\right) .
$$

Here $i, l, t \geq 0$ and $j, s \geq 1$. In particular, $f_{1}$ is a chain map of complexes. $f$ is an $A_{\infty}$-quasiisomorphism if $f_{1}$ induces identities on all cohomologies. $f$ is strict if $f_{n}=0$ for all $n \geq 2$. We will identify $f$ with $f_{1}$ in this case.

Proposition 3.2. ([14, Proposition 3.3.1.7]) Let $A$ be a strictly unital $A_{\infty}$-algebra over $R$ and $M$ be a strictly unital $A_{\infty}$-module over $A$. Then there is a strictly unital minimal $A_{\infty}$-module over $A$ which is $A_{\infty}$-quasi-isomorphic to $M$. 
3.4. Derived categories. Let $A$ be a strictly unital $A_{\infty}$-algebra over $R$. Let $\operatorname{Mod}_{\infty}(A)$ be the category of strictly unital $A_{\infty}$-modules over $A$ with strictly unital $A_{\infty}$-morphisms as morphisms. The derived category $\mathcal{D}(A)$ is the category obtained from $\operatorname{Mod}_{\infty}(A)$ by formally inverting all $A_{\infty}$-quasi-isomorphisms. The category $\mathcal{D}(A)$ is a triangulated category whose suspension functor is the shift functor $\Sigma$. It has arbitrary (set-indexed) direct sums.

Let $A$ be a dg algebra over $R$. There are three classes of modules we can consider. First we can view $A$ as a strictly unital $A_{\infty}$-algebra over $R$ with vanishing $m_{n}$ for $n \geq 3$ and consider strictly unital $A_{\infty}$-modules over $A$. Secondly, we can consider unital dg $A$-modules (see [14, Section 2.1.1]), which are exactly the strictly unital $A_{\infty}$-modules $M$ over $A$ with $m_{n}^{M}=0$ for $n \geq 3$. Thirdly, we can consider $\mathrm{dg}$ modules over $A$ which is considered a dg $k$-algebra. It is easy to check that the second and the third classes coincide. By [14, Lemme 4.1.3.8], the derived category of $\operatorname{dg} A$-modules is canonically equivalent to the derived category of strictly unital $A_{\infty}$-modules over $A$. We will identify these two derived categories.

Theorem 3.3. ([14, Théorème 4.1.2.4]) Let $f: A \rightarrow B$ be a strictly unital $A_{\infty}$-quasi-isomorphism of strictly unital $A_{\infty}$-algebras over $R$. Then there is a triangle equivalence $f^{*}: \mathcal{D}(B) \rightarrow \mathcal{D}(A)$ which takes $B_{B}$ to an $A_{\infty}$-module isomorphic to $A_{A}$ in $\mathcal{D}(A)$.

3.5. Morphisms from 'projectives'. Let $A$ be a strictly unital $A_{\infty}$-algebra over $R$. Let $e$ be a strict idempotent of $A$. For a strictly unital $A_{\infty}$-module $M$ over $A$ let $M e=\{m e:=$ $\left.m_{2}^{M}(m \otimes e) \mid m \in M\right\}$. Then applying the identity (3.3) for $n=2$ to $m \otimes e$, we get

$$
m_{1}(m e)=(-1)^{|m|} m m_{1}(e)+m_{1}(m) e=m_{1}(m) e \in M e .
$$

So $M e$ is a subcomplex of $M$. We will need the following result.

Lemma 3.4. Let e be a strict idempotent of $A$. For a strictly unital $A_{\infty}$-module $M$ over $A$ and an integer $p$, there is an isomorphism

$$
\operatorname{Hom}_{\mathcal{D}(A)}\left(e A, \Sigma^{p} M\right) \cong H^{p}(M e)
$$

Proof. This can be obtained as a consequence of a suitable version of Yoneda's lemma. We do not find a reference in the literature, so we give a direct proof here. It is enough to prove for the case $p=0$. By [14, Théorème 4.1.3.1], $\operatorname{Hom}_{\mathcal{D}(A)}(e A, M)$ is the same as the space of strictly unital $A_{\infty}$-morphisms from $e A$ to $M$ modulo those homotopic to 0 . We show in four steps that this space is canonically isomorphic to $H^{0}(M e)$. Here two strictly unital $A_{\infty}$-morphisms $f, g: M \rightarrow M^{\prime}$ of strictly unital $A_{\infty}$-modules are homotopic if there is a strictly unital homotopy $h$ between $f$ and $g$, that is, a family of homogeneous $K$-linear maps $h_{n}: M \otimes_{R} A^{\otimes_{R}(n-1)} \rightarrow M^{\prime}$ $(n \geq 1)$ of degree $-n$ such that $h_{n}\left(\operatorname{id}_{M} \otimes \mathrm{id} \otimes \cdots \otimes \mathrm{id} \otimes \eta \otimes \mathrm{id} \otimes \cdots \otimes \mathrm{id}\right)=0$ for all $n \geq 2$ and that the following identity holds for all $n \geq 1$

$$
f_{n}-g_{n}=\sum_{s+t=n}(-1)^{t} m_{1+t}\left(h_{s} \otimes \mathrm{id}^{\otimes t}\right)+\sum_{i+j+l=n}(-1)^{i j+l} h_{i+1+l}\left(\mathrm{id}^{\otimes i} \otimes m_{j} \otimes \mathrm{id}^{\otimes l}\right) .
$$


Step 1: Let $m \in Z^{0}(M e)$. For $n \geq 1$, define a homogeneous $R$-linear map of degree $1-n$

$$
\begin{aligned}
& f_{n}: e A \otimes_{R} A^{\otimes_{R}(n-1)} \longrightarrow M \\
& a_{1} \otimes a_{2} \otimes \cdots \otimes a_{n} \longmapsto(-1)^{n+1} m_{n+1}\left(m \otimes a_{1} \otimes \cdots \otimes a_{n}\right) .
\end{aligned}
$$

Then $f=\left(f_{n}\right)_{n \geq 1}$ is a strictly unital $A_{\infty}$-morphism from $e A$ to $M$.

The identity $f_{n}\left(\mathrm{id}_{e A} \otimes \mathrm{id} \otimes \cdots \otimes \mathrm{id} \otimes \eta \otimes \mathrm{id} \otimes \cdots \otimes \mathrm{id}\right)=0(n \geq 2)$ is clear since $A$ is strictly unital. We need to check the identity (3.4) for all $n \geq 1$ applied to $a_{1} \otimes a_{2} \otimes \cdots a_{n}$, where $a_{1} \in e A$ and $a_{2}, \ldots, a_{n} \in A$ are homogenous. We have (note that when flipping tensors we have the Koszul sign: $\left.(\varphi \otimes \psi)(u \otimes v)=(-1)^{|\psi| \cdot|u|}(\varphi(u) \otimes \psi(v))\right)$

$$
\begin{aligned}
\text { LHS }= & \sum_{i+j+l=n}(-1)^{i j+l} f_{i+1+l}\left((-1)^{\left(\left|a_{1}\right|+\ldots+\left|a_{i}\right|\right)(2-j)} a_{1} \otimes \cdots \otimes a_{i}\right. \\
& \left.\otimes m_{j}\left(a_{i+1} \otimes \cdots \otimes a_{i+j}\right) \otimes a_{i+j+1} \otimes \cdots \otimes a_{n}\right) \\
= & \sum_{i+j+l=n}(-1)^{i j+l}(-1)^{i+2+l} m_{i+2+l}\left((-1)^{\left(\left|a_{1}\right|+\ldots+\left|a_{i}\right|\right)(2-j)} m \otimes a_{1} \otimes \cdots \otimes a_{i}\right. \\
& \left.\otimes m_{j}\left(a_{i+1} \otimes \cdots \otimes a_{i+j}\right) \otimes a_{i+j+1} \otimes \cdots \otimes a_{n}\right) \\
= & \sum_{i+j+l=n+1, i \geq 1}(-1)^{i j+l+n} m_{i+1+l}\left(\mathrm{id}^{\otimes i} \otimes m_{j} \otimes \mathrm{id}^{\otimes l}\right)\left(m \otimes a_{1} \otimes \cdots \otimes a_{n}\right) \\
\mathrm{RHS}= & \sum_{s+t=n} m_{1+t}\left(f_{s}\left(a_{1} \otimes \cdots \otimes a_{s}\right) \otimes a_{s+1} \otimes \cdots \otimes a_{n}\right) \\
= & \sum_{s+t=n}(-1)^{s+1} m_{1+t}\left(m_{s+1}\left(m \otimes a_{1} \otimes \cdots \otimes a_{s}\right) \otimes a_{s+1} \otimes \cdots \otimes a_{n}\right) \\
= & \sum_{s+t=n+1, s \geq 2}(-1)^{n+1+t} m_{1+t}\left(m_{s} \otimes \mathrm{id}^{\otimes t}\right)\left(m \otimes a_{1} \otimes \cdots \otimes a_{n}\right) .
\end{aligned}
$$

By (3.3), we have

$$
\begin{aligned}
\mathrm{LHS}-\mathrm{RHS} & =-m_{1+n}\left(m_{1} \otimes \mathrm{id}^{\otimes n}\right)\left(m \otimes a_{1} \otimes \cdots \otimes a_{n}\right) \\
& =-m_{1+n}\left(m_{1}(m) \otimes a_{1} \otimes \cdots \otimes a_{n}\right) \\
& =0 .
\end{aligned}
$$

Step 2: Let $m \in Z^{0}(M e)$. In Step 1, we associate to $m$ a strictly unital $A_{\infty}$-morphism $f$ from $e A$ to $M$. We claim that $f$ is homotopic to 0 if and only if $m$ belongs to $B^{0}(M e)$.

We first show that for any $m \in M e$ we have $m=m e$. Suppose $m=m^{\prime} e$. Applying (3.3) for $n=3$ to $m^{\prime} \otimes e \otimes e$ we get

$$
m=m^{\prime} e=m^{\prime}(e e)=\left(m^{\prime} e\right) e=m e .
$$

Now we prove the 'only if' part. Assume that $f$ is homotopic to 0 . Then there exists a homogeneous $R$-linear map $h_{1}: e A \rightarrow M$ of degree -1 such that $f_{1}=m_{1} h_{1}+h_{1} m_{1}$. So

$$
m=m e=f_{1}(e)=m_{1} h_{1}(e)+h_{1} m_{1}(e)=m_{1} h_{1}(e) \in B^{0}(M e),
$$

as $e$ is a strict idempotent. 
Next we prove the 'if' part. Assume that $m=m_{1}\left(m^{\prime}\right)$, where $m^{\prime} \in M^{-1}$. For $n \geq 1$, define a homogeneous $R$-linear map of degree $-n$

$$
\begin{aligned}
& h_{n}: e A \otimes_{R} A^{\otimes_{R}(n-1)} \longrightarrow M \\
& \quad a_{1} \otimes a_{2} \otimes \cdots \otimes a_{n} \longmapsto m_{n+1}\left(m^{\prime} \otimes a_{1} \otimes \cdots \otimes a_{n}\right) .
\end{aligned}
$$

Then $h=\left(h_{n}\right)_{\geq 1}$ is a strictly unital homotopy between $f$ and 0 . The identity $h_{n}\left(\mathrm{id}_{e A} \otimes \mathrm{id} \otimes\right.$ $\cdots \otimes \mathrm{id} \otimes \eta \otimes \mathrm{id} \otimes \cdots \otimes \mathrm{id})=0(n \geq 2)$ is clear since $A$ is strictly unital. We need to check the identity (3.6) for all $n \geq 1$ applied to $a_{1} \otimes a_{2} \otimes \cdots \otimes a_{n}$, where $a_{1} \in e A$ and $a_{2}, \ldots, a_{n} \in A$ are homogeneous. We have

$$
\begin{aligned}
\mathrm{RHS}= & \sum_{s+t=n}(-1)^{t} m_{1+t}\left(h_{s}\left(a_{1} \otimes \cdots \otimes a_{s}\right) \otimes a_{s+1} \otimes \cdots \otimes a_{n}\right) \\
& +\sum_{i+j+l=n}(-1)^{i j+l} h_{i+1+l}\left((-1)^{\left(\left|a_{1}\right|+\ldots+\left|a_{i}\right|\right)(2-j)} a_{1} \otimes \cdots \otimes a_{i}\right. \\
& \left.\otimes m_{j}\left(a_{i+1} \otimes \cdots \otimes a_{i+j}\right) \otimes a_{i+j+1} \otimes \cdots \otimes a_{n}\right) \\
= & \sum_{s+t=n}(-1)^{t} m_{1+t}\left(m_{s+1}\left(m^{\prime} \otimes a_{1} \otimes \cdots \otimes a_{s}\right) \otimes a_{s+1} \otimes \cdots \otimes a_{n}\right) \\
& +\sum_{i+j+l=n}(-1)^{(i+1) j+l} m_{i+2+l}\left((-1)^{\left(\left|m^{\prime}\right|+\left|a_{1}\right|+\ldots+\left|a_{i}\right|\right)(2-j)} m^{\prime} \otimes a_{1} \otimes \cdots \otimes a_{i}\right. \\
= & \left.\sum_{s+t=n+1, s \geq 2}(-1)^{t} m_{1+t}\left(m_{s} \otimes \mathrm{id}^{\otimes t}\right)\right)\left(m^{\prime} \otimes a_{1} \otimes \cdots \otimes a_{n}\right) \\
& +\sum_{i+j+l=n+1, i \geq 1}(-1)^{i j+l} m_{i+1+l}\left(\mathrm{id}^{\otimes i} \otimes m_{j} \otimes \mathrm{id}^{\otimes l}\right)\left(m^{\prime} \otimes a_{1} \otimes \cdots \otimes a_{n}\right) \\
& \stackrel{(3.3)}{=}-(-1)^{n} m_{1+n}\left(m_{1} \otimes \mathrm{id}^{\otimes n}\right)\left(m^{\prime} \otimes a_{1} \otimes \cdots \otimes a_{n}\right) \\
= & (-1)^{n+1} m_{n+1}\left(m \otimes a_{1} \otimes \cdots \otimes a_{n}\right) \\
= & f_{n}\left(a_{1} \otimes \cdots \otimes a_{n}\right)=\mathrm{LHS}_{i+j} .
\end{aligned}
$$

Step 3: Let $f$ be a strictly unital $A_{\infty}$-morphism from $e A$ to $M$. We first show that $f_{1}(e) \in$ $Z^{0}(M e)$. The identity (3.4) for $n=1$ applied to $e$ yields $m_{1}\left(f_{1}(e)\right)=f_{1}\left(m_{1}(e)\right)=0$, so $f_{1}(e) \in Z^{0}(M)$. The same identity for $n=2$ applied to $e \otimes e$ yields $f_{1}(e)=f_{1}(e) e+m_{1} f_{2}(e \otimes e)=$ $f_{1}(e) e \in M e$. The last equality holds because $f_{2}(\mathrm{id} \otimes \eta)=0$ and $e \in \operatorname{im}(\eta)$.

We claim that $f$ is homotopic to the strictly unital $A_{\infty}$-morphism from $e A$ to $M$ associated to $f_{1}(e)$ as in Step 1. It is enough to show that if $f_{1}(e)=0$, then $f$ is homotopic to 0 .

For $n \geq 1$, define a homogeneous $R$-linear map of degree $-n$

$$
\begin{aligned}
& h_{n}: e A \otimes_{R} A^{\otimes_{R}(n-1)} \longrightarrow M \\
& \quad a_{1} \otimes a_{2} \otimes \cdots \otimes a_{n} \longmapsto(-1)^{n+1} f_{n+1}\left(e \otimes a_{1} \otimes \cdots \otimes a_{n}\right) .
\end{aligned}
$$

Then $h=\left(h_{n}\right)_{n \geq 1}$ is a strictly unital homotopy between $f$ and 0 . The identity $h_{n}\left(\mathrm{id}_{e A} \otimes \mathrm{id} \otimes\right.$ $\cdots \otimes \mathrm{id} \otimes \eta \otimes \mathrm{id} \otimes \cdots \otimes \mathrm{id})=0(n \geq 2)$ is clear since $f$ is strictly unital. We need to check the 
identity (3.6) for all $n \geq 1$ applied to $a_{1} \otimes a_{2} \otimes \cdots \otimes a_{n}$, where $a_{1} \in e A$ and $a_{2}, \ldots, a_{n} \in A$ are homogeneous. We have

$$
\begin{aligned}
& \mathrm{RHS}=\sum_{s+t=n}(-1)^{t} m_{1+t}\left(h_{s}\left(a_{1} \otimes \cdots \otimes a_{s}\right) \otimes a_{s+1} \otimes \cdots \otimes a_{n}\right) \\
& +\sum_{i+j+l=n}(-1)^{i j+l} h_{i+1+l}\left((-1)^{\left(\left|a_{1}\right|+\ldots+\left|a_{i}\right|\right)(2-j)} a_{1} \otimes \cdots \otimes a_{i}\right. \\
& \left.\otimes m_{j}\left(a_{i+1} \otimes \cdots \otimes a_{i+j}\right) \otimes a_{i+j+1} \otimes \cdots \otimes a_{n}\right) \\
& =\sum_{s+t=n}(-1)^{t} m_{1+t}\left((-1)^{s+1} f_{s+1}\left(e \otimes a_{1} \otimes \cdots \otimes a_{s}\right) \otimes a_{s+1} \otimes \cdots \otimes a_{n}\right) \\
& +\sum_{i+j+l=n}(-1)^{i j+l}(-1)^{i+2+l} f_{i+2+l}\left((-1)^{\left(\left|a_{1}\right|+\ldots+\left|a_{i}\right|\right)(2-j)} e \otimes a_{1} \otimes \cdots \otimes a_{i}\right. \\
& \left.\otimes m_{j}\left(a_{i+1} \otimes \cdots \otimes a_{i+j}\right) \otimes a_{i+j+1} \otimes \cdots \otimes a_{n}\right) \\
& \left.=\sum_{s+t=n+1, s \geq 2}(-1)^{n+1} m_{1+t}\left(f_{s} \otimes \mathrm{id}^{\otimes t}\right)\right)\left(e \otimes a_{1} \otimes \cdots \otimes a_{n}\right) \\
& +\sum_{i+j+l=n}(-1)^{(i+1) j+l+n} f_{i+2+l}\left(\mathrm{id}^{\otimes(i+1)} \otimes m_{j} \otimes \mathrm{id}^{\otimes l}\right)\left(e \otimes a_{1} \otimes \cdots \otimes a_{n}\right) \\
& \left.\stackrel{f_{1}(e)=0}{=} \sum_{s+t=n+1}(-1)^{n+1} m_{1+t}\left(f_{s} \otimes \mathrm{id}^{\otimes t}\right)\right)\left(e \otimes a_{1} \otimes \cdots \otimes a_{n}\right) \\
& +\sum_{i+j+l=n+1, i \geq 1}(-1)^{i j+l+n} f_{i+1+l}\left(\mathrm{id}^{\otimes i} \otimes m_{j} \otimes \mathrm{id}^{\otimes l}\right)\left(e \otimes a_{1} \otimes \cdots \otimes a_{n}\right) \\
& \stackrel{\text { 3.4. }}{=}-(-1)^{n} \sum_{j+l=n+1}(-1)^{l} f_{1+l}\left(m_{j} \otimes \mathrm{id}^{\otimes l}\right)\left(e \otimes a_{1} \otimes \cdots \otimes a_{n}\right) \\
& =f_{n}\left(m_{2} \otimes \mathrm{id}^{\otimes n}\right)\left(e \otimes a_{1} \otimes \cdots \otimes a_{n}\right) \\
& =f_{n}\left(a_{1} \otimes \cdots \otimes a_{n}\right)=\text { LHS. }
\end{aligned}
$$

Step 4: By Step 3, $\operatorname{Hom}_{\mathcal{D}(A)}(e A, M)$ is canonically isomorphic to the space of strictly unital $A_{\infty}$-morphisms from $e A$ to $M$ associated to $m \in Z^{0}(M e)$ modulo those homotopic to 0 , and hence to $H^{0}(M e)=Z^{0}(M e) / B^{0}(M e)$ by Step 2 .

3.6. Perfect derived categories and finite-dimensional derived categories. Denote by $\operatorname{per}(A)$ the thick subcategory of $\mathcal{D}(A)$ generated by $A_{A}$, and denote by $\mathcal{D}_{f d}(A)$ the full subcategory of $\mathcal{D}(A)$ consisting of those $A_{\infty}$-modules whose total cohomology is finite-dimensional.

Lemma 3.5. (a) Let $M$ be an object of $\mathcal{D}(A)$. Then $M$ is compact if and only if it belongs to $\operatorname{per}(A)$, and $M$ belongs to $\mathcal{D}_{f d}(A)$ if and only if $\bigoplus_{i \in \mathbb{Z}} \operatorname{Hom}_{\mathcal{D}(A)}\left(N, \Sigma^{i} M\right)$ is finitedimensional for any $N \in \operatorname{per}(A)$.

(b) Let $A$ and $B$ be two strictly unital $A_{\infty}$-algebras over $R$. $A$ triangle equivalence $\mathcal{D}(A) \rightarrow$ $\mathcal{D}(B)$ restricts to triangle equivalences $\operatorname{per}(A) \rightarrow \operatorname{per}(B)$ and $\mathcal{D}_{f d}(A) \rightarrow \mathcal{D}_{f d}(B)$.

Proof. Let $A^{\prime}$ be a dg model of $A$ as in Theorem 3.1. Then by Theorem 3.3 there is a triangle equivalence $\mathcal{D}\left(A^{\prime}\right) \rightarrow \mathcal{D}(A)$ which takes $A_{A^{\prime}}^{\prime}$ to $A_{A}$. By [11, Corollary 3.7] (also 9, Remark 5.3 (a)]), an object of $\mathcal{D}\left(A^{\prime}\right)$ is compact if and only if it belongs to $\operatorname{per}\left(A^{\prime}\right)$. The first assertion 
of (a) follows immediately. The second assertion of (a) follows from Lemma 3.4 by dévissage. (b) is a direct consequence of (a) since $\operatorname{per}(A)$ and $\mathcal{D}_{f d}(A)$ admit intrinsic descriptions inside $\mathcal{D}(A)$.

3.7. Morita theorems. We have the following Morita theorems for derived categories and for algebraic triangulated categories. They can be considered as special cases of [14, Théorème 7.6.0.4] and [14, Théorème 7.6.0.6], respectively.

Theorem 3.6. Let $A$ be a strictly unital $A_{\infty}$-algebra over $R$. Let $\left\{X_{1}, \ldots, X_{r}\right\}$ be a set of compact generators of $\mathcal{D}(A)$, i.e. $\operatorname{per}(A)=\operatorname{thick}\left(X_{1}, \ldots, X_{r}\right)$. Let $K$ be the direct product of $r$ copies of $k$. Then there is a strictly unital minimal $A_{\infty}$-algebra $B$ over $K$ such that as a graded algebra

$$
B=\bigoplus_{p \in \mathbb{Z}} \operatorname{Hom}_{\mathcal{D}(A)}\left(\bigoplus_{i=1}^{r} X_{i}, \Sigma^{p} \bigoplus_{i=1}^{r} X_{i}\right),
$$

and there is a triangle equivalence

$$
\mathcal{D}(B) \longrightarrow \mathcal{D}(A)
$$

taking $e_{i} B(1 \leq i \leq r)$ to $X_{i}$.

Proof. By [14, Théorème 7.6.0.4], there is a strictly unital minimal $A_{\infty}$-category $\mathcal{B}$ whose objects are $X_{1}, \ldots, X_{r}$, and whose morphism spaces are $\operatorname{Hom}_{\mathcal{B}}\left(X_{i}, X_{j}\right)=\bigoplus_{p \in \mathbb{Z}} \operatorname{Hom}_{\mathcal{D}(\mathcal{A})}\left(X_{i}, \Sigma^{p} X_{j}\right)$ for $1 \leq i, j \leq r$, and a triangle equivalence

$$
\mathcal{D}(\mathcal{B}) \longrightarrow \mathcal{D}(A)
$$

which takes the free $A_{\infty}$-module $X_{i}^{\wedge}$ associated to the object $X_{i} \in \mathcal{B}$ to $X_{i} \in \mathcal{D}(A)$ for $1 \leq i \leq r$. We identify $K$ with $k\left\{\operatorname{id}_{X_{1}}\right\} \times \cdots \times k\left\{\operatorname{id}_{X_{r}}\right\}$. Let $B$ be the graded $K$-bimodule $\bigoplus_{i, j=1}^{r} \bigoplus_{p \in \mathbb{Z}} \operatorname{Hom}_{\mathcal{D}(\mathcal{A})}\left(X_{i}, \Sigma^{p} X_{j}\right)$. Then the multiplications on $\mathcal{B}$ induce multiplications on $B$ such that $B$ becomes a minimal strictly unital $A_{\infty}$-algebra over $K$. The assignment $M \mapsto$ $\bigoplus_{i=1}^{r} M\left(X_{i}\right)$ extends to an isomorphism $\operatorname{Mod}_{\infty}(\mathcal{B}) \rightarrow \operatorname{Mod}_{\infty}(B)$, which induces a triangle isomorphism $\mathcal{D}(\mathcal{B}) \rightarrow \mathcal{D}(B)$. So we have the desired triangle equivalence.

Theorem 3.7. Let $\mathcal{C}$ be an idempotent complete algebraic triangulated category. Assume that $\mathcal{C}$ is generated by a set of objects $\left\{X_{1}, \ldots, X_{r}\right\}$, i.e. $\mathcal{C}=\operatorname{thick}\left(X_{1}, \ldots, X_{r}\right)$. Let $K$ be the direct product of $r$ copies of $k$. Then there is a strictly unital minimal $A_{\infty}$-algebra $B$ over $K$ such that as a graded algebra

$$
B=\bigoplus_{p \in \mathbb{Z}} \operatorname{Hom}_{\mathcal{C}}\left(\bigoplus_{i=1}^{r} X_{i}, \Sigma^{p} \bigoplus_{i=1}^{r} X_{i}\right),
$$

and there is a triangle equivalence

$$
\operatorname{per}(B) \longrightarrow \mathcal{C}
$$

taking $e_{i} B(1 \leq i \leq r)$ to $X_{i}$.

Proof. This is a consequence of [14, Théorème 7.6.0.6]. The proof is similar to that of the preceding result. 


\section{Simple-minded collections and minimal positive $A_{\infty}$-Algebras}

In this section we recall the definition of simple-minded collections and study strictly unital minimal positive $A_{\infty}$-algebras, which are closely related to simple-minded collections.

4.1. Simple-minded collections. Let $\mathcal{C}$ be a triangulated category with suspension functor $\Sigma$. A collection $\left\{X_{1}, \ldots, X_{r}\right\}$ of objects of $\mathcal{C}$ is simple-minded if

- $\operatorname{Hom}_{\mathcal{C}}\left(X_{i}, \Sigma^{p} X_{j}\right)=0, \forall p<0$ and $1 \leq i, j \leq r$,

- $\operatorname{Hom}_{\mathcal{C}}\left(X_{i}, X_{j}\right)=0$ if $1 \leq i \neq j \leq r$ and $\operatorname{End}_{\mathcal{C}}\left(X_{i}\right)$ is a division $k$-algebra for all $1 \leq i \leq r$, - $\mathcal{C}=\operatorname{thick}\left(X_{1}, \ldots, X_{r}\right)$.

Two simple-minded collections $\left\{X_{1}, \ldots, X_{r}\right\}$ and $\left\{X_{1}^{\prime}, \ldots, X_{r}^{\prime}\right\}$ are said to be isomorphic if up to reordering we have $X_{i} \cong X_{i}^{\prime}$ for all $1 \leq i \leq r$. A simple-minded collection $\left\{X_{1}, \ldots, X_{r}\right\}$ is said to be elementary if $\operatorname{End}_{\mathcal{C}}\left(X_{i}\right) \cong k$ for all $1 \leq i \leq r$. If the field $k$ is algebraically closed, then every simple-minded collection in $\mathcal{C}$ is elementary. In general $\mathcal{C}$ may contain non-elementary simple-minded collections and it is not known whether any two simple-minded collections in $\mathcal{C}$ have the same set of endomorphism algebras. It is the case when the two simple-minded collections are related by a mutation ([13, Remark 7.7]).

Let $A$ be a non-positive dg $k$-algebra with $H^{0}(A)$ being finite-dimensional over $k$. Then a complete set of pairwise non-isomorphic simple $H^{0}(A)$-modules, viewed as $\operatorname{dg} A$-modules via the homomorphism $A \rightarrow H^{0}(A)$, form a simple-minded collection in $\mathcal{D}_{f d}(A)$, see for example [6. Theorem A.1 (c)].

4.2. Strictly unital minimal positive $A_{\infty}$-algebras. Fix $r \in \mathbb{N}$. Let $K$ be the direct product of $r$ copies of $k$. Let $e_{1}, \ldots, e_{r}$ be the standard basis of $K$ over $k$.

Let $A$ be a strictly unital minimal $A_{\infty}$-algebra over $K$. We say that $A$ is positive if

$-A^{p}=0$ for all $p<0$,

- $A^{0}=K$ and the unit is the embedding $K=A^{0} \hookrightarrow A$.

It is clear that $e_{1}, \ldots, e_{r}$ are strict idempotents of $A$. So $e_{1} A, \ldots, e_{r} A$ are submodules of $A$. Moreover, as an $A_{\infty}$-module $A=\bigoplus_{i=1}^{r} e_{i} A$.

Lemma 4.1. (a) Let $A$ be a strictly unital minimal positive $A_{\infty}$-algebra over $K$. Then $\left\{e_{1} A, \ldots, e_{r} A\right\}$ is an elementary simple-minded collection in $\operatorname{per}(A)$.

(b) Let $\mathcal{C}$ be an idempotent complete algebraic triangulated category and let $\left\{X_{1}, \ldots, X_{r}\right\}$ be an elementary simple-minded collection in $\mathcal{C}$. Then there is a strictly unital minimal positive $A_{\infty}$-algebra $A$ over $K$ together with a triangle equivalence $\mathcal{C} \rightarrow \operatorname{per}(A)$ which takes $X_{i}(1 \leq i \leq r)$ to $e_{i} A$.

Proof. (a) Put $P_{i}=e_{i} A(1 \leq i \leq r)$. Then by Lemma 3.4 we have

- $\operatorname{Hom}\left(P_{i}, \Sigma^{p} P_{j}\right)=H^{p}\left(e_{j} A e_{i}\right)=0$ for $p<0$,

. $\operatorname{Hom}\left(P_{i}, P_{j}\right)=H^{0}\left(e_{j} A e_{i}\right)= \begin{cases}k & \text { if } i=j, \\ 0 & \text { otherwise. }\end{cases}$ 
Moreover, $P_{1}, \ldots, P_{r}$ generates $\operatorname{per}(A)$ since $A=P_{1} \oplus \ldots \oplus P_{r}$. Therefore $P_{1}, \ldots, P_{r}$ is an elementary simple-minded collection in $\operatorname{per}(A)$.

(b) By Theorem 3.7 there is a strictly unital minimal $A_{\infty}$-algebra $A$ over $K$ such that as a graded algebra

$$
A=\bigoplus_{p \in \mathbb{Z}} \operatorname{Hom}_{\mathcal{C}}\left(\bigoplus_{i=1}^{r} X_{i}, \Sigma^{p} \bigoplus_{i=1}^{r} X_{i}\right),
$$

and there is a triangle equivalence

$$
\mathcal{C} \longrightarrow \operatorname{per}(A)
$$

taking $X_{i}$ to $e_{i} A, 1 \leq i \leq r$. That $A$ is positive follows from the assumption that $\left\{X_{1}, \ldots, X_{r}\right\}$ is an elementary simple-minded collection.

Let $A$ be a strictly unital minimal positive $A_{\infty}$-algebra over $K$. The projection $\varepsilon: A \rightarrow$ $A^{0}=K$ makes $A$ an augmented $A_{\infty}$-algebra over $K$. We view $K$ as an $A_{\infty}$-module over $A$ via $\varepsilon$ and denote it by $S$. For $1 \leq i \leq r$, we have a 1-dimensional $A_{\infty}$-module $S_{i}=e_{i} A / e_{i} \operatorname{ker}(\varepsilon)$. Then $S=S_{1} \oplus \ldots \oplus S_{r}$. We call $S_{1}, \ldots, S_{r}$ the simple modules over $A$. Let $M$ be a strictly unital $A_{\infty}$-module over $A$ which is concentrated in degree 0 . Then for all $m \in M$ we have $m_{n}^{M}\left(m \otimes a_{1} \otimes \cdots \otimes a_{n-1}\right)=0$ if $a_{1}, \ldots, a_{n-1}$ are homogenous and one of them belongs to $\operatorname{ker}(\varepsilon)$. Indeed, we may assume that $m \in M^{0}$. If at least one of $a_{1}, \ldots, a_{n-1}$ belongs to $A^{0}$ and at least one of them belongs to $\operatorname{ker}(\varepsilon)$, then $m_{n}^{M}\left(m \otimes a_{1} \otimes \cdots \otimes a_{n-1}\right)=0$ because $M$ is strictly unital. If all $a_{1}, \ldots, a_{n-1}$ belong to $\operatorname{ker}(\varepsilon)$, then $m_{n}^{M}\left(m \otimes a_{1} \otimes \cdots \otimes a_{n-1}\right)$ is homogeneous of degree different from 0 and has to be zero. So the $A_{\infty}$-module structure on $M$ factors through $\varepsilon$, so $M$ is the direct sum of copies of simple modules.

Lemma 4.2. Let $M$ be a strictly unital $A_{\infty}$-module over $A$. Fix $1 \leq i \leq r$. If for $1 \leq j \leq r$ and $p \in \mathbb{Z}$ we have

$$
\operatorname{Hom}_{\mathcal{D}(A)}\left(e_{j} A, \Sigma^{p} M\right)= \begin{cases}k & \text { if } j=i \text { and } p=0 \\ 0 & \text { otherwise }\end{cases}
$$

then $M$ is $A_{\infty}$-quasi-isomorphic to $S_{i}$.

Proof. By Lemma 3.4, we have

$$
H^{p}\left(M e_{j}\right)= \begin{cases}k & \text { if } j=i \text { and } p=0 \\ 0 & \text { otherwise }\end{cases}
$$

So by Proposition 3.2, $M$ is $A_{\infty}$-quasi-isomorphic to a strictly unital $A_{\infty}$-module $M^{\prime}$, which is 1-dimensional and concentrated in degree 0 . Moreover $M^{\prime} e_{i}=k$ and $M^{\prime} e_{j}=0$ for $j \neq i$. So $M^{\prime}$ is isomorphic to $S_{i}$.

The first statement of the following lemma, phrased in terms of dg algebras and dg modules, can be obtained by combining [12, Lemma 5.2] and [1, Step 6 of the proof of Lemma 4.8]. 
Lemma 4.3. The $A_{\infty}$-module $S$ is a silting object in $\mathcal{D}_{f d}(A) \sqrt{1}$. Moreover, if $A$ is Koszul as a graded algebra (see for example [4] for a definition), then $S$ is a tilting object in $\mathcal{D}_{f d}(A)$.

Proof. We first show that $\operatorname{Hom}_{\mathcal{D}(A)}\left(S, \Sigma^{m} S\right)=0$ for $m>0$. Take the enveloping algebra $U$ of $A$ as in Theorem 3.1. Then $U$ is an augmented dg algebra over $K$ and there is a strictly unital $A_{\infty}$-quasi-isomorphism $A \rightarrow U$ of augmented $A_{\infty}$-algebras over $K$. So $S$ can be viewed as a dg $U$-module and the $A_{\infty}$-structure on $S$ over $A$ factors through the $A_{\infty}$-quasi-isomorphism $A \rightarrow U$. By Theorem 3.3, there is a triangle equivalence $\mathcal{D}(U) \rightarrow \mathcal{D}(A)$ which sends $S$ to $S$. So we only need to show that $\operatorname{Hom}_{\mathcal{D}(U)}\left(S, \Sigma^{m} S\right)=0$ for $m>0$. We view $S$ as a graded module over the graded algebra $H^{*}(U)$, which is identified with $A$ viewed as a graded algebra. $S$ admits a projective resolution over the graded algebra $A$

$$
\ldots \rightarrow P^{m} \rightarrow P^{m+1} \rightarrow \ldots \rightarrow P^{-1} \rightarrow P^{0}
$$

such that $P^{m} \in \operatorname{Add}\left(A\left\langle m^{\prime}\right\rangle \mid m^{\prime} \leq m\right)$, where $\langle 1\rangle$ denotes the degree shift. According to [9, Theorem 3.1 (c)], there is a $\operatorname{dg}$ module $P$ over $U$ which is quasi-isomorphic to $S$ and which admits a filtration

$$
0=F_{-1} \subset F_{0} \subset \ldots \subset F_{p} \subset F_{p+1} \subset \ldots \subset P, p \in \mathbb{N}
$$

such that

(F1) $P$ is the union of the $F_{p}, p \in \mathbb{N}$.

(F2) $\forall p \in \mathbb{N}$, the inclusion morphism $F_{p-1} \subset F_{p}$ splits in the category Grmod $U$ of graded modules over $U$, which is considered as a graded algebra by forgetting the differential.

(F3) $\forall p \in \mathbb{N}, F_{p} / F_{p-1} \in \operatorname{Add}\left(\Sigma^{m} A \mid m \leq 0\right)$.

By (F1) and (F2), we have an isomorphism $P \cong \bigoplus_{p \geq 0} F_{p} / F_{p-1}$ in Grmod $U$. So as a graded vector space $\mathcal{H o m} U(P, S)=\prod_{p \geq 0} \mathcal{H o m} U\left(F_{p} / F_{p-1}, S\right)$, which is concentrated in non-positive degrees by (F3), since $\mathcal{H} o m_{U}\left(\Sigma^{m} U, S\right)=\Sigma^{-m} S$. As a consequence, we obtain that for $m>0$

$$
\operatorname{Hom}_{\mathcal{D}(U)}\left(S, \Sigma^{m} S\right)=\operatorname{Hom}_{\mathcal{D}(U)}\left(P, \Sigma^{m} S\right)=H^{m} \mathcal{H} m_{U}(P, S)=0 .
$$

Next we show that $\mathcal{D}_{f d}(A)=\operatorname{thick}(S)$. By Proposition 3.2, it suffices to prove that if a strictly unital minimal $A_{\infty}$-module $M$ over $A$ satisfies that $\operatorname{dim}(M):=\bigoplus_{m \in \mathbb{Z}} M^{m}$ is finitedimensional, then $M \in \operatorname{thick}(S)$. Up to shift we may assume that $M^{m}=0$ for all $m<0$ and

\footnotetext{
${ }^{1}$ At first sight this statement may seem surprising. There are two typical situations: (1) the dg algebra/ $A_{\infty}$ algebra is non-positive and the extensions between simple modules are in positive degrees, see the last paragraph of Section 4.1 (2) the dg algebra/ $A_{\infty}$-algebra is positive (this means that there are extensions in positive degrees between the 'projective modules'! See Lemma 4.1) and the extensions between simple modules are in non-positive degrees. Morally the first-order extensions between simple modules are dual to generators up to a degree shift. For example take the graded algebra $A=k[x]$ and the simple module $S=A /(x)$. Then apart from the scalar endomorphisms there are 1-dimensional self-extensions of $S$, say with basis $y$. Then $|y|=1-|x|$. In particular, if the degree of $x$ changes from $-\infty$ to $+\infty$, then the degree of $y$ changes from $+\infty$ to $-\infty$ (as if there is a mirror at $\left.\frac{1}{2}\right)$.
} 
$M^{0} \neq 0$. Define $M^{>0}=\bigoplus_{m>0} M^{m}$. Then $M^{>0}$ is an submodule of $M$. Let $\iota: M^{>0} \rightarrow M$ be the embedding and form a triangle in $\mathcal{D}(A)$

$$
M^{>0} \stackrel{\iota}{\longrightarrow} M \longrightarrow \bar{M} \longrightarrow \Sigma M^{>0} .
$$

Here $\bar{M}$ is assumed to be minimal. Looking at the long exact sequence of cohomologies, we see that $\bar{M}$ is concentrated in degree 0 , and hence is a finite direct sum of copies of $S_{1}, \ldots, S_{r}$. Now by induction on $\operatorname{dim}(M)$ we finish the proof.

Finally, assume that $A$ is Koszul. Then the above resolution of $S$ can be chosen such that $P^{m} \in \operatorname{Add}(A\langle m\rangle)$. Consequently, $F_{p} / F_{p-1} \in \operatorname{Add}(U)$ and $\mathcal{H} o m_{U}(P, S)$ is concentrated in degree 0. It follows that $\operatorname{Hom}_{\mathcal{D}(U)}\left(S, \Sigma^{m} S\right)=0$ for $m \neq 0$.

\section{Constructing Silting objeCts From Simples-Minded COlleCtions}

In this section we will use Koszul duality to construct a silting object in the perfect derived category of a finite-dimensional non-positive dg algebra from a given simple-minded collection in the finite-dimensional derived category .

Let $A$ be a non-positive dg $k$-algebra whose total cohomology is finite-dimensional over $k$.

5.1. The standard simple-minded collection. Note that $H^{0}(A)$ is a finite-dimensional algebra. Let $S_{1}, \ldots, S_{r}$ be a complete set of pairwise non-isomorphic simple $H^{0}(A)$-modules and view them as $\operatorname{dg} A$-modules via the homomorphism $A \rightarrow H^{0}(A)$. Recall that $\left\{S_{1}, \ldots, S_{r}\right\}$ is a simple-minded collection in $\mathcal{D}_{f d}(A)$. We assume further that $\operatorname{End}_{H^{0}(A)}\left(S_{i}\right) \cong k$ for all $1 \leq i \leq r$. Then $\left\{S_{1}, \ldots, S_{r}\right\}$ is an elementary simple-minded collection in $\mathcal{D}_{f d}(A)$. We will see in the proof of Theorem 5.5 that every elementary simple-minded collection in $\mathcal{D}_{f d}(A)$ is of this form up to derived equivalence.

Since $\operatorname{End}_{\mathcal{D}(A)}(A)=H^{0}(A)$, it follows that the functor $H^{0}=\operatorname{Hom}_{\mathcal{D}(A)}(A$, ?) restricts to an equivalence $\operatorname{add}_{\mathcal{D}(A)}(A) \stackrel{\simeq}{\rightarrow} \operatorname{proj} H^{0}(A)$. Therefore there are indecomposable objects $P_{1}, \ldots, P_{r} \in \operatorname{add}_{\mathcal{D}(A)}(A) \subseteq \operatorname{per}(A)$ such that $H^{0}\left(P_{1}\right), \ldots, H^{0}\left(P_{r}\right)$ are projective covers of $S_{1}, \ldots, S_{r}$, respectively. In particular, there are positive integers $a_{1}, \ldots, a_{r}$ such that $A \cong$ $\bigoplus_{i=1}^{r} P_{i}^{\oplus a_{i}}$ in $\mathcal{D}(A)$. Moreover, for $p \neq 0$, the space $\operatorname{Hom}_{\mathcal{D}(A)}\left(P_{i}, \Sigma^{p} S_{j}\right)$ vanishes as it is a direct summand of $\operatorname{Hom}_{\mathcal{D}(A)}\left(A, \Sigma^{p} S_{j}\right)=H^{p}\left(S_{j}\right)=0$. For $p=0$, consider the triangle

$$
\sigma^{\leq-1}\left(P_{i}\right) \rightarrow P_{i} \rightarrow H^{0}\left(P_{i}\right) \rightarrow \Sigma \sigma^{\leq-1}\left(P_{i}\right),
$$

where $\sigma^{\leq-1}$ is the standard truncation of complexes at degree -1 . Applying $\operatorname{Hom}_{\mathcal{D}(A)}\left(?, S_{j}\right)$ to this triangle, we obtain a long exact sequence

$\operatorname{Hom}_{\mathcal{D}(A)}\left(\Sigma \sigma^{\leq-1}\left(P_{i}\right), S_{j}\right) \rightarrow \operatorname{Hom}_{\mathcal{D}(A)}\left(H^{0}\left(P_{i}\right), S_{j}\right) \rightarrow \operatorname{Hom}_{\mathcal{D}(A)}\left(P_{i}, S_{j}\right) \rightarrow \operatorname{Hom}_{\mathcal{D}(A)}\left(\sigma^{\leq-1}\left(P_{i}\right), S_{j}\right)$

By [6, Theorem A.1 (b)], the two outer terms vanish and $\operatorname{Hom}_{\mathcal{D}(A)}\left(H^{0}\left(P_{i}\right), S_{j}\right)$ is isomorphic to $\operatorname{Hom}_{H^{0}(A)}\left(H^{0}\left(P_{i}\right), S_{j}\right)$. So $\operatorname{Hom}_{\mathcal{D}(A)}\left(P_{i}, S_{j}\right) \cong \operatorname{Hom}_{H^{0}(A)}\left(H^{0}\left(P_{i}\right), S_{j}\right)$ vanishes if $i \neq j$ and is isomorphic to $k$ if $i=j$. To sum up, we have

$$
\operatorname{Hom}_{\mathcal{D}(A)}\left(P_{i}, \Sigma^{p} S_{j}\right)= \begin{cases}k & \text { if } i=j \text { and } p=0 \\ 0 & \text { otherwise. }\end{cases}
$$


Further, the collection $\left\{S_{1}, \ldots, S_{r}\right\}$ is determined by this property. Namely, fix $1 \leq j \leq r$ and let $M \in \mathcal{D}(A)$ be such that

$$
\operatorname{Hom}_{\mathcal{D}(A)}\left(P_{i}, \Sigma^{p} M\right)= \begin{cases}k & \text { if } i=j \text { and } p=0 \\ 0 & \text { otherwise }\end{cases}
$$

then $M \cong S_{j}$ in $\mathcal{D}(A)$. Indeed, this property implies that $H^{p}(M) \cong \operatorname{Hom}_{\mathcal{D}(A)}\left(A, \Sigma^{p} M\right)$ is trivial unless $p=0$, so $M$ is isomorphic in $\mathcal{D}(A)$ to $H^{0}(M)$, which is a $\operatorname{dg} A$-module via the homomorphism $A \rightarrow H^{0}(A)$. Moreover, applying $\operatorname{Hom}_{\mathcal{D}(A)}\left(\right.$ ?,$\left.H^{0}(M)\right)$ to the triangle (5.1) we obtain

$$
\operatorname{Hom}_{H^{0}(A)}\left(H^{0}\left(P_{i}\right), H^{0}(M)\right) \cong \operatorname{Hom}_{\mathcal{D}(A)}\left(P_{i}, H^{0}(M)\right)= \begin{cases}k & \text { if } i=j, \\ 0 & \text { otherwise. }\end{cases}
$$

It follows that $H^{0}(M) \cong S_{j}$ in $\bmod H^{0}(A)$. Therefore $M \cong S_{j}$ in $\mathcal{D}(A)$.

Let $I_{i}=\nu\left(P_{i}\right)$ for $1 \leq i \leq r$. Then $D\left({ }_{A} A\right)=\bigoplus_{i=1}^{r} I_{i}^{\oplus a_{i}}$ and by the Auslander-Reiten formula we have

$$
\operatorname{Hom}_{\mathcal{D}(A)}\left(S_{i}, \Sigma^{p} I_{j}\right)= \begin{cases}k & \text { if } i=j \text { and } p=0 \\ 0 & \text { otherwise. }\end{cases}
$$

Let $K$ be the direct sum of $r$ copies of $k$ and let $e_{1}, \ldots, e_{r}$ be the standard basis of $K$ over $k$. By Lemma 4.1 (b), there is a strictly unital minimal positive $A_{\infty}$-algebra $\mathcal{S}$ over $K$ such that as a graded algebra

$$
\mathcal{S}=\bigoplus_{p \in \mathbb{Z}} \operatorname{Hom}_{\mathcal{D}_{f d}(A)}\left(\bigoplus_{i=1}^{r} S_{i}, \Sigma^{p} \bigoplus_{i=1}^{r} S_{j}\right)
$$

and there is a triangle equivalence

$$
\Phi: \mathcal{D}_{f d}(A) \longrightarrow \operatorname{per}(\mathcal{S})
$$

taking $S_{i}(1 \leq i \leq r)$ to $e_{i} \mathcal{S}$. Therefore we have

$$
\operatorname{Hom}_{\mathcal{D}(\mathcal{S})}\left(e_{i} \mathcal{S}, \Sigma^{p} \Phi\left(I_{j}\right)\right)= \begin{cases}k & \text { if } i=j \text { and } p=0 \\ 0 & \text { otherwise. }\end{cases}
$$

By Lemma 4.2, $\Phi\left(I_{1}\right), \ldots, \Phi\left(I_{r}\right)$ are, up to $A_{\infty}$-quasi-isomorphism, precisely the simple modules over $\mathcal{S}$. In other words, the equivalence $\Phi$ restricts to a triangle equivalence

$$
\Phi \mid: \operatorname{thick}_{\mathcal{D}(A)}\left(D\left({ }_{A} A\right)\right)=\operatorname{thick}_{\mathcal{D}(A)}\left(I_{1}, \ldots, I_{r}\right) \longrightarrow \operatorname{thick}_{\mathcal{D}(\mathcal{S})}\left(\Phi\left(I_{1}\right), \ldots, \Phi\left(I_{r}\right)\right)=\mathcal{D}_{f d}(\mathcal{S}),
$$

where the last equality follows from Lemma 4.3, It follows that $\mathcal{D}_{f d}(\mathcal{S}) \subseteq \operatorname{per}(\mathcal{S})$.

5.2. Construction of the silting object. Let $\left\{X_{1}, \ldots, X_{r}\right\} \subseteq \mathcal{D}_{f d}(A)$ be an elementary simple-minded collection. Let $Y_{i}=\Phi\left(X_{i}\right)$ for $1 \leq i \leq r$. Then $\left\{Y_{1}, \ldots, Y_{r}\right\}$ is an elementary simple-minded collection in $\operatorname{per}(\mathcal{S})$. By Theorem 3.6, there is a strictly unital minimal positive $A_{\infty}$-algebra $\mathcal{X}$ over $K$ such that as a graded algebra

$$
\mathcal{X}=\bigoplus_{p \in \mathbb{Z}} \operatorname{Hom}_{\mathcal{D}_{f d}(A)}\left(\bigoplus_{i=1}^{r} Y_{i}, \Sigma^{p} \bigoplus_{i=1}^{r} Y_{i}\right)
$$


and there is a triangle equivalence

$$
\tilde{\Psi}: \mathcal{D}(\mathcal{S}) \longrightarrow \mathcal{D}(\mathcal{X})
$$

taking $Y_{i}(1 \leq i \leq r)$ to $e_{i} \mathcal{X}$. By Lemma 3.5, $\tilde{\Psi}$ restricts to triangle equivalences

$$
\begin{gathered}
\Psi: \operatorname{per}(\mathcal{S}) \longrightarrow \operatorname{per}(\mathcal{X}), \\
\Psi \mid: \mathcal{D}_{f d}(\mathcal{S}) \longrightarrow \mathcal{D}_{f d}(\mathcal{X}) .
\end{gathered}
$$

This implies that $\mathcal{D}_{f d}(\mathcal{X}) \subseteq \operatorname{per}(\mathcal{X})$. So we have the following commutative diagram

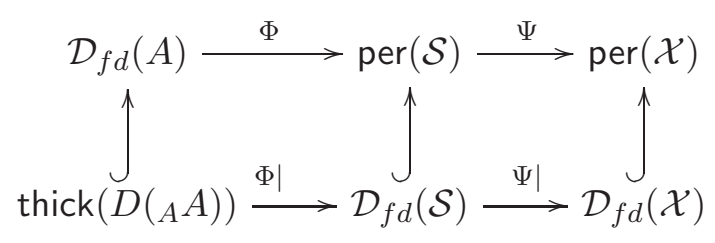

Let $R_{1}, \ldots, R_{r}$ be the simple modules over $\mathcal{X}$, and let $T_{1}, \ldots, T_{r}$ be their images under a quasi-inverse of the equivalence $(\Psi \circ \Phi) \mid$. Put $T=\bigoplus_{i=1}^{r} T_{i}$.

Proposition 5.1. $\quad$ (a) $T$ is a silting object of thick $\left(D\left({ }_{A} A\right)\right)$.

(b) For $1 \leq i, j \leq r$, and $p \in \mathbb{Z}$,

$$
\operatorname{Hom}_{\mathcal{D}_{f d}(A)}\left(X_{j}, \Sigma^{p} T_{i}\right)= \begin{cases}k & \text { if } i=j \text { and } p=0, \\ 0 & \text { otherwise. }\end{cases}
$$

(c) $\nu^{-1} T$ is a silting object of $\operatorname{per}(A)$.

(d) For $1 \leq i, j \leq r$, and $m \in \mathbb{Z}$,

$$
\operatorname{Hom}_{\mathcal{D}_{f d}(A)}\left(\nu^{-1} T_{i}, \Sigma^{p} X_{j}\right)= \begin{cases}k & \text { if } i=j \text { and } p=0 \\ 0 & \text { otherwise. }\end{cases}
$$

Proof. (a) This is because $R_{1} \oplus \ldots \oplus R_{r}$ is a silting object of $\mathcal{D}_{f d}(\mathcal{X})$ (Lemma4.3) and $(\Psi \circ \Phi)$ is a triangle equivalence.

(b) By Lemma 3.4 we have

$$
\operatorname{Hom}\left(e_{j} \mathcal{X}, \Sigma^{p} R_{i}\right)= \begin{cases}k & \text { if } i=j \text { and } p=0, \\ 0 & \text { otherwise. }\end{cases}
$$

The desired formula follows immediately because $\Psi \circ \Phi$ is a triangle equivalence.

(c) follows from (a) because $\nu: \operatorname{per}(A) \rightarrow \operatorname{thick}\left(D\left({ }_{A} A\right)\right)$ is a triangle equivalence.

(d) follows from (b) and the Auslander-Reiten formula.

If $A$ is a finite-dimensional elementary (ordinary) $k$-algebra, then this is 13 , Lemmas 5.6, 5.7 and 5.8 and Proposition 5.9], up to the hypothesis that if $\mathcal{D}^{b}(\bmod A)=\mathcal{D}_{f d}(A)$ has an elementary simple-minded collection, then all simple-minded collections in $\mathcal{D}^{b}(\bmod A)$ are elementary. Compared with Rickard's construction [18] used in [13], our new approach has the disadvantage that it may fail for non-elementary simple-minded collections, but it also has some advantages. By Lemma 4.3, we obtain a sufficient condition on $\nu^{-1} T$ being a tilting object. 
Corollary 5.2. If $\mathcal{X}$ as a graded algebra is Koszul, then $\nu^{-1} T$ is a tilting object of $\operatorname{per}(A)$.

By [20], if $\mathcal{X}$ as a graded algebra is isomorphic to $k Q$, where $Q$ is a graded quiver with all arrows in positive degrees, then $\mathcal{D}_{f d}(A)$ is triangle equivalent to $\operatorname{per}(k Q)$, where $k Q$ is considered as a dg algebra with trivial differential. If all arrows of $Q$ are in degree 1 , then it is known that $\operatorname{per}(k Q)$ is triangle equivalent to the radical-square-zero algebra $R$ associated to the opposite quiver $Q^{o p}$, considered as an ungraded quiver, see for example [7, Theorem 2.5]. Consequently, $A$ is derived equivalent to $R$.

Furthermore, the dg endomorphism algebra and the truncated dg endomorphism algebra $\tilde{\Gamma}$ of $\nu^{-1} T$ (see Section 2.3) are Koszul dual to the $A_{\infty}$-algebra $\mathcal{X}$. So they can be obtained, up to quasi-equivalence (in the sense of [9, Section 7]), as the dual bar construction of $\mathcal{X}$ : the complete tensor algebra $B^{\#} \mathcal{S}=\widehat{T}_{K}\left(D\left(\mathcal{X}^{>0}[1]\right)\right)$ of $D\left(\mathcal{X}^{>0}[1]\right)$ over $K$ (see [15, 8]), where $\mathcal{X}>0=\bigoplus_{p>0} \mathcal{X}^{p}$. In other words, up to quasi-equivalence the following diagram is commutative

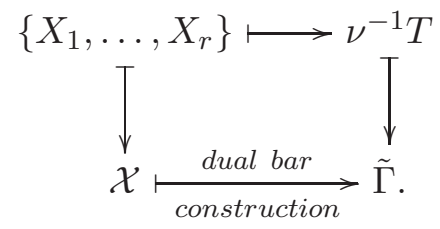

In general the $A_{\infty}$-structure on $\mathcal{X}$ is hard to compute. However, sometimes it is easy to obtain the $A_{\infty}$-structure on the truncated part $\mathcal{X}^{[0,2]}$, the $A_{\infty}$-algebra obtained from $\mathcal{X}$ by modulo the elements of degree $\geq 3$. We have

$$
\operatorname{End}_{\mathcal{D}(A)}\left(\nu^{-1} T\right)=H^{0}\left(\mathcal{E} n d_{A}\left(\nu^{-1} T\right)\right)=H^{0}\left(B^{\#} \mathcal{X}\right)=H^{0}\left(B^{\#} \mathcal{X}^{[0,2]}\right)
$$

The quiver $Q$ of $\operatorname{End}_{\mathcal{D}(A)}\left(\nu^{-1} T\right)$ is determined by the $K$-bimodule structure on $\mathcal{X}^{1}$. Precisely, the set of vertices of $Q$ is $\{1, \ldots, r\}$, and the number of arrows from $i$ to $j$ is the dimension of $e_{i} \mathcal{X}^{1} e_{j}$ over $k$. The relations of $\operatorname{End}_{\mathcal{D}(A)}\left(\nu^{-1} T\right)$ are 'dual' to the restrictions $m_{n}:\left(\mathcal{X}^{1}\right)^{\otimes_{K} n} \rightarrow$ $\mathcal{X}^{2}$ of the multiplications of $\mathcal{X}$.

Corollary 5.3. If $\mathcal{X}^{1}=0$, then as an algebra $\operatorname{End}_{\mathcal{D}(A)}\left(\nu^{-1} T\right)$ is isomorphic to $K$.

The following example is taken from [3]. Let $A$ be the algebra given by the quiver $1 \underset{\beta}{\stackrel{\alpha}{\rightleftarrows}} 2$ with relations $\alpha \beta=0=\beta \alpha$. Take $X_{1}=P_{1}$ and $X_{2}=\Sigma^{-1} S_{1}$. The $\mathcal{X}$ as a graded algebra is the path algebra of the graded quiver

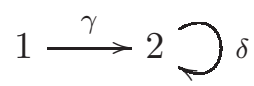

where $\gamma$ is of degree 1 and $\delta$ is of degree 2. Simply because of lack of morphisms to multiply with, the $A_{\infty}$-structure on $\mathcal{X}^{[0,2]}$ is trivial. The dual bar construction shows that $\operatorname{End}_{\mathcal{D}(A)}\left(\nu^{-1} T\right)$ is the path algebra of the ungraded quiver $1 \longleftarrow 2$. 
5.3. Main results. Now we state our main result, which is a consequence of Proposition 5.1,

Theorem 5.4. For an elementary simple-minded collection $\left\{X_{1}, \ldots, X_{r}\right\}$ of $\mathcal{D}_{f d}(A)$, there exists a unique (up to isomorphism) silting object $M=M_{1} \oplus \ldots \oplus M_{r}$ of $\operatorname{per}(A)$ such that for $1 \leq i, j \leq r$ and $p \in \mathbb{Z}$

$$
\operatorname{Hom}_{\mathcal{D}_{f d}(A)}\left(M_{i}, \Sigma^{p} X_{j}\right)= \begin{cases}k & \text { if } i=j \text { and } p=0 \\ 0 & \text { otherwise. }\end{cases}
$$

Proof. The existence of $M$ follows from Proposition 5.1 (c)(d): take $M=\nu^{-1} T$. Let $N=$ $N_{1} \oplus \ldots \oplus N_{r}$ be an object of $\operatorname{per}(A)$ such that for $1 \leq i, j \leq r$ and $p \in \mathbb{Z}$

$$
\operatorname{Hom}_{\mathcal{D}_{f d}(A)}\left(N_{i}, \Sigma^{p} X_{j}\right)= \begin{cases}k & \text { if } i=j \text { and } p=0, \\ 0 & \text { otherwise. }\end{cases}
$$

Then by the Auslander-Reiten formula we have

$$
\operatorname{Hom}_{\mathcal{D}_{f d}(A)}\left(\Sigma^{p} X_{j}, \nu N_{i}\right)= \begin{cases}k & \text { if } i=j \text { and } p=0 \\ 0 & \text { otherwise. }\end{cases}
$$

Applying the triangle equivalence $\Psi \circ \Phi$ we obtain

$$
\operatorname{Hom}_{\mathcal{D}_{f d}(A)}\left(\Sigma^{p} e_{j} \mathcal{X}, \Psi \circ \Phi \circ \nu\left(N_{i}\right)\right)= \begin{cases}k & \text { if } i=j \text { and } p=0 \\ 0 & \text { otherwise. }\end{cases}
$$

Therefore by Lemma 4.2 we have $\Psi \circ \Phi \circ \nu\left(N_{i}\right) \cong R_{i}$. So $N_{i} \cong \nu^{-1} \circ(\Psi \circ \Psi)^{-1}\left(R_{i}\right)=M_{i}$, showing the uniqueness of $M$.

As a consequence, we obtain the following theorem, generalising [13, Theorems 6.1 and 7.12]. The assumption that $k$ is algebraically closed is required to ensure that all simpleminded collections are elementary, which is used only in the construction of the map from simple-minded collections to silting objects.

Theorem 5.5. Assume that $k$ is algebraically closed. Then there are one-to-one correspondences which commute with mutations and which preserve partial orders between

(1) equivalence classes of silting objects in $\operatorname{per}(A)$,

(2) isomorphism classes of simple-minded collections in $\mathcal{D}_{f d}(A)$,

(3) bounded $t$-structures of $\mathcal{D}_{f d}(A)$ with length heart,

(4) bounded co-t-structures of $\operatorname{per}(A)$.

Proof. The proof is the same as that of [13, Theorems 6.1 and 7.12]. Here we only give the definition of some of the correspondences, which are compatible with each other.

From silting objects to simple-minded collections: Let $M$ be a basic silting object in $\operatorname{per}(A)$.We may assume that $M$ is $K$-projective. Let $\tilde{\Gamma}$ be the truncated dg endomorphism algebra of $M$ (see Section 2.3). Then $\tilde{\Gamma}$ is non-positive and has finite-dimensional total cohomology; moreover, there is a triangle equivalence $\mathcal{D}(\tilde{\Gamma}) \rightarrow \mathcal{D}(A)$ taking $\tilde{\Gamma}$ to $M$. The simple-minded collection $\left\{X_{1}, \ldots, X_{r}\right\}$ corresponding to $M$ is the image of a complete collection of pairwise 
non-isomorphic simple $H^{0}(\tilde{\Gamma})$-modules (viewed as dg $\tilde{\Gamma}$-modules) under this equivalence. It is the unique collection (up to isomorphism) in $\mathcal{D}_{f d}(A)$ satisfying for $1 \leq i, j \leq r$ and $p \in \mathbb{Z}$

$$
\operatorname{Hom}\left(M_{i}, \Sigma^{p} X_{j}\right)= \begin{cases}k & \text { if } i=j \text { and } p=0 \\ 0 & \text { otherwise. }\end{cases}
$$

From silting objects to t-structures: Let $M$ be a silting object in $\operatorname{per}(A)$. The corresponding $t$-structure $\left(\mathcal{D}^{\leq 0}, \mathcal{D}^{\geq 0}\right)$ on $\mathcal{D}_{f d}(A)$ is defined as

$$
\begin{aligned}
& \mathcal{D}^{\leq 0}=\left\{X \in \mathcal{D}_{f d}(A) \mid \operatorname{Hom}\left(M, \Sigma^{p} X\right)=0 \text { for } p>0\right\}, \\
& \mathcal{D}^{\geq 0}=\left\{X \in \mathcal{D}_{f d}(A) \mid \operatorname{Hom}\left(M, \Sigma^{p} X\right)=0 \text { for } p<0\right\} .
\end{aligned}
$$

This is the image of the standard $t$-structure $\left(\left[\underline{6}\right.\right.$, Theorem A.1]) on $\mathcal{D}_{f d}(\tilde{\Gamma})$ under the triangle equivalence $\mathcal{D}_{f d}(\tilde{\Gamma}) \rightarrow \mathcal{D}_{f d}(A)$ (which is restricted from the triangle equivalence $\mathcal{D}(\tilde{\Gamma}) \rightarrow \mathcal{D}(A)$ ). The heart of this t-structure is equivalent to $\bmod \operatorname{End}(M)$.

From silting objects to co-t-structures: Let $M$ be a silting object in $\operatorname{per}(A)$. The corresponding co- $t$-structure $\left(\mathcal{P}_{\geq 0}, \mathcal{P}_{\leq 0}\right)$ on $\operatorname{per}(A)$ is defined as (see [13, Section 3.4])

$$
\begin{aligned}
& \mathcal{P}_{\geq 0}=\text { the smallest full subcategory of } \operatorname{per}(A) \text { which contains }\left\{\Sigma^{p} M \mid p \leq 0\right\} \\
& \text { and which is closed under taking extensions and direct summands, } \\
& \mathcal{P}_{\leq 0}=\text { the smallest full subcategory of } \operatorname{per}(A) \text { which contains }\left\{\Sigma^{p} M \mid p \geq 0\right\} \\
& \text { and which is closed under taking extensions and direct summands. }
\end{aligned}
$$

From $t$-structures to simple-minded collections: Let $\left(\mathcal{D}^{\leq 0}, \mathcal{D}^{\geq 0}\right)$ be a bounded $t$-structure on $\mathcal{D}_{f d}(A)$ with length heart. The corresponding simple-minded collection is a complete collection of pairwise non-isomorphic simple objects of the heart $\mathcal{D}^{\leq 0} \cap \mathcal{D}^{\geq 0}$ (see [13, Section 3.3]).

From simple-minded collections to silting objects: Let $\left\{X_{1}, \ldots, X_{r}\right\}$ be a simple-minded collection of $\mathcal{D}_{f d}(A)$. It is elementary since the base field $k$ is algebraically closed. The corresponding silting object is the $M$ as in Theorem 5.4

From co-t-structures to silting objects: Let $\left(\mathcal{P}_{\geq 0}, \mathcal{P}_{\leq 0}\right)$ be a bounded co-t-structure on $\operatorname{per}(A)$. The corresponding silting object $M$ of $\operatorname{per}(A)$ is an additive generator of the co-heart, i.e. $\operatorname{add}(M)=\mathcal{P}_{\geq 0} \cap \mathcal{P}_{\leq 0}$ (see [13, Sections 3.1 and 3.4]).

\section{REFERENCES}

[1] Takahide Adachi, Yuya Mizuno and Dong Yang, Silting-discrete triangulated categories and contractible stability spaces, arXiv:1708.08168v1.

[2] Takuma Aihara and Osamu Iyama, Silting mutation in triangulated categories, J. London Math. Soc. 85 (2012), no. 3, 633-668.

[3] Salah Al-Nofayee, Simple objects in the heart of a t-structure, J. Pure Appl. Algebra 213 (2009), no. 1, 54-59.

[4] Alexander Beilinson, Victor Ginzburg, and Wolfgang Soergel, Koszul duality patterns in representation theory, J. Amer. Math. Soc. 9 (1996), no. 2, 473-527.

[5] Mikhail V. Bondarko, Weight structures vs. t-structures; weight filtrations, spectral sequences, and complexes (for motives and in general), J. K-Theory 6 (2010), no. 3, 387-504. 
[6] Thomas Brüstle and Dong Yang, Ordered exchange graphs, Advances in Representation Theory of Algebras, 135-193, EMS Ser. Congr. Rep., Eur. Math. Soc., Zürich, 2013.

[7] Xiao-Wu Chen and Dong Yang, Homotopy categories, Leavitt path algebras and GP Modules, International Mathematics Research Notices, Vol. 2015, No. 10, 2597-2633.

[8] Martin Kalck and Dong Yang, Relative singularity categories I: Auslander resolutions, Adv. Math. 301 (2016), 973-2021.

[9] Bernhard Keller, Deriving DG categories, Ann. Sci. École Norm. Sup. (4) 27 (1994), no. 1, 63-102.

[10] _ Introduction to A-infinity algebras and modules, Homology Homotopy Appl. 3 (2001), no. 1, 1-35.

[11] _ On differential graded categories, International Congress of Mathematicians. Vol. II, Eur. Math. Soc., Zürich, 2006, pp. 151-190.

[12] Bernhard Keller and Pedro Nicolás, Weight structures and simple dg modules for positive dg algebras, Int. Math. Res. Not. IMRN 2013, no. 5, 1028-1078.

[13] Steffen Koenig and Dong Yang, Silting objects, Simple-minded collections, t-structures and co-t-structures for finite-dimensional algebras, Doc. Math. 19 (2014), 403-438.

[14] Kenji Lefèvre-Hasegawa, Sur les $A_{\infty}$-catégories, Thèse de doctorat, Université Denis Diderot - Paris 7 , November 2003, arXiv:math.CT/0310337.

[15] Di Ming Lu, John H. Palmieri, Quan Shui Wu, and James J. Zhang, Koszul equivalences in $A_{\infty}$-algebras, New York J. Math. 14 (2008), 325-378.

[16] Valery A. Lunts, Formality of DG algebras (after Kaledin), J. Algebra 323 (2010), no. 4, 878-898.

[17] Octavio Mendoza, Edith C. Sáenz, Valente Santiago, and María José Souto Salorio, Auslander-Buchweitz context and co-t-structures, Appl. Categor. Struct. 21 (2013), 417-440.

[18] _ Equivalences of derived categories for symmetric algebras, J. Algebra 257 (2002), no. 2, 460-481.

[19] Jeremy Rickard and Raphael Rouquier, Stable categories and reconstruction, J. Algebra (2016), 10.1016/j.jalgebra.2016.05.018.

[20] Hao Su, A note on path $A_{\infty}$-algebras over positively graded quivers, preprint (2016), arXiv:1601.04309.

Hao Su, School of Mathematical Sciences, University of Science and Technology of China, Hefei, Anhui 230026, P. R. China

E-mail address: suhao@mail.ustc.edu.cn

Dong Yang, Department of Mathematics, Nanjing University, Nanjing 210093, PR China

E-mail address: yangdong@nju.edu.cn 